\title{
Invited commentary on ... Mental health research priorities in low- and middle-income countries ${ }^{\dagger}$
}

\author{
Ricardo Araya
}

\begin{abstract}
Summary
There are huge inequalities in health research within and between countries. It is argued that this may hinder the process of setting and tackling mental health priorities. If this were true, establishing research priorities would be important. However, this is not a simple process and one must be aware of its limitations. Despite a plethora of declarations, funding for mental health research
\end{abstract}

in low- and middle-income countries remains hard to find. In the absence of funding, establishing research priorities is seen by many as an exercise of lesser importance.

\section{Declaration of interest}

None.
Sharan et $\mathrm{l}^{1}$ (this issue) state that there is a paucity and lack of prioritisation of mental health research in low- and middleincome (LAMI) countries. There is no doubt that little research is conducted in LAMI countries: the ' $9 / 10$ gap divide' in health research has become an established slogan depicting the hideous inequalities between countries.

Nine out of 10 publications in peer-reviewed journals come from high-income countries, where only $10 \%$ of the world's population live. We must not forget that there are also marked inequalities in health research within LAMI countries. So these odious inequalities are not all due to the selfish high-income countries but also to the self-interested ruling minorities in LAMI countries. To address the lack of prioritisation, the authors embarked on an exercise to establish research priorities seeking the opinion of thousands of experts from 114 LAMI countries.

\section{The importance of research to decision-making}

It is likely, although not certain by any means, that LAMI countries may benefit from more and better research in mental health. Most experts will argue that research is essential to improve health systems. It can lead to the development of more cost-effective services and a better understanding of the causes of ill health. Decisions on future investments in health, if there is such a thing, need to be supported by sound research evidence. In the absence of this research, some may argue, it is difficult to make those decisions. Since resources are so limited it may be imperative to know what research priorities are there.

Although the previous arguments are logical, all those who have worked in policy-making in LAMI countries know that social and political factors are often more important than research evidence. Thus, some people may argue that it is better to devote scarce resources to treating people rather than undertaking research whose value in influencing decision-making would be limited. Research investment is so restricted that it may not even merit the effort of setting priorities. Moreover, even when science comes up with some interesting finding this often does not reach or influence policy-makers. Researchers often fail to understand that scientific information must be actively rather than passively spread. ${ }^{2}$ If mental health research is so important, why is it that

†See pp. 354-363, this issue. so few LAMI governments are investing so little? Why have so few funding bodies seen this as a priority?

\section{How much can we believe in studies establishing research priorities?}

Establishing research priorities is a complex pursuit. Different methodologies have been used and all have advantages and disadvantages, as the authors argue. Their study was based on a fairly large survey of researchers and stakeholders from a wide range of LAMI countries. This is a major strength of this paper. Often, mental health research priorities for LAMI countries are decided upon by international experts with little knowledge or experience working in any of these settings. It is also of merit that stakeholders were invited to give an opinion. The conclusions are similar to those deriving from the Lancet Mental Health series, which used a similar methodology. It is reassuring to see some concordance between these two exercises but both were affected by similar problems and both may have reached the same but wrong conclusions. It is also reassuring to see that there is some good agreement between researchers and stakeholders and across countries and regions, giving a sense of global unity to these priorities. The authors did a great job highlighting all the limitations of the study but, as they state, it is a first step that must be improved in future iterations. Finally, it is also encouraging to see that those neuropsychiatric conditions that have the highest attached health burden were also given the highest priority. A recent editorial ${ }^{3}$ complained that often there is no consistency in how health and health research priorities are perceived and funded.

One limitation of this study, as well as of similar exercises, is the difficulty of ascertaining the representativeness of the sample. The authors attempted to achieve a target number but it is unclear how many were approached and what proportion replied. Thus, it is possible that the responses represent a small and biased group of experts, among whom I include myself. Why did so few researchers return their questionnaires? The authors give some possible reasons. In my opinion an important reason is that there is a sense of 'combat fatigue' in many LAMI countries when it comes to mental health research. In spite of a stream of carefully worded declarations, international conferences, and movements and organisations being assembled, too little funding is coming 
forward for mental health research or programmes. Many of the experts approached may have thought it was yet another futile exercise in the absence of fresh resources. What would be the point of setting priorities for something unlikely to lead to action? There are examples of programmes, for instance the Grand Challenges in Global Health initiative (www.gcgh.org), where there was an upfront commitment of US $\$ 200$ million (later increased to $\$ 450$ million) in research funding. One wonders what kind of response would be achieved if there were an offer of injecting a substantial amount of fresh resources for mental health research.

Another important limitation of this, as well as other similar exercises, is that clinicians and patients were not included in the consultation. The general tendency is to consult researchers, as in this study. It is no surprise that often those delivering services on the ground feel that research priorities are far removed from what is needed most urgently to improve the lives of people with mental illness. The pre-fixed question format may have also limited the depth in the answers, something acknowledged by the authors. An exercise using a different methodology to identify the grand challenges and research priorities for chronic diseases delivered a much more precise set of research questions that should take prominence. ${ }^{4}$ It is not enough to know that epidemiological studies and health systems research were ranked highest on types of needed research. We need to know what the big questions requiring urgent answers are. Some innovative ways of establishing priorities, such as asking people how they would invest money, have been used in other exercises. For instance, an exercise to establish the single intervention that would most help to improve the health of those living in poverty was conducted. Most of the people interviewed were public health experts but it is interesting to note that not a single person mentioned mental health. It is important to remember that resources for mental health research are allocated in competition with other health research priorities and in this respect we are not doing very well either. $^{5}$

Finally, it is useful to reflect upon this idea of grouping countries with low or middle incomes as if they represent a unitary concept. This is an untested assumption and we must recognise that there are wide variations between countries belonging to any of these income bands. Given the widespread acceptance of these classification systems it is not surprising to find out that some experts may think that because an intervention proved to be effective in Argentina, there is no need to test it in Nigeria. The concept of 'one size fits all' is handy but rather simplistic and possibly counterproductive.
What can really make a difference for mental health research in LAMI countries?

It goes without saying that the most important thing is to increase the funding and resources for mental health research in LAMI countries. The health burden of non-communicable diseases is growing rapidly in LAMI countries and it will become a future priority; mental health will be competing for funding. It is likely that there will be more funding in the future. The newly emerging mega-economies, such as Brazil, India and China, are also beginning to invest larger sums in their own research and there is some hope that their research findings may be more relevant to other, less-developed nations. We therefore need to prepare the ground to attract this funding and make the best possible use of it. However, funding is not the only problem. A large enough critical mass of mental health researchers in LAMI countries is still an aspiration. According to some experts, the lack of career paths to attract and retain good researchers is the most serious impediment to health research in Africa. ${ }^{6}$ Priority-setting is and will be a subject of controversy especially in view of the inherent limitations and complexities. Nonetheless, more can be gained than lost in engaging in these exercises for setting mental health research priorities. The sooner more of us understand this, the better for the advancement of the cause.

Ricardo Araya, MRCPsych, University of Bristol, Division of Psychiatry, Cotham House Cotham Hill, Bristol BS6 6JL, UK. Email: R.Araya@bristol.ac.uk

First received 22 Apr 2009, final revision 23 Jun 2009, accepted 22 Jul 2009

\section{References}

1 Sharan P, Gallo C, Gureje O, Lamberte E, Mari JJ, Mazzotti G, et al. Mental health research prioirities in low- and middle-income countries of Africa, Asia, Latin America and the Caribbean. Br J Psychiatry 2009; 195: 354-63.

2 McCannon C, Berwick D, Massoud M. The science of large-scale change in global health. JAMA 2007; 298: 1937-9.

3 Lopez A. Health and health-research priorities: has WHO got it right? Lancet 2008; 372: 1525-7.

4 Daar A, Singer P, Persad D, Pramming SG, Matthews DR, Beaglehole R, et al. Grand challenges in chronic noncommunicable diseases. Nature 2007; 450: 494-6.

5 Gamey $Y$. Which single intervention would do the most to improve the health of those living on less than \$1 per day? PLOS Med 2007; 4: e303.

6 Whitworth J, Kokwaro G, Kinyanjui S, Snewin, VA, Tanner M, Walport M, et al. Strengthening capacity for health research in Africa. Lancet 2008; 372: $1590-3$. 